\title{
Effects of reaction temperature on size and optical properties of CdSe nanocrystals
}

\author{
SHUTANG CHEN, XIAOLING ZHANG*, YANBING ZHAO and QIUHUA ZHANG \\ Department of Chemistry, School of Science, Beijing Institute of Technology, Beijing 100081, P.R. China
}

MS received 15 March 2009; revised 6 July 2009

\begin{abstract}
We report experimental results on the reaction temperature dependence of luminescence properties in size-controlled CdSe nanocrystals. Such reaction temperature dependent property is also sizedependent. The diameter of the CdSe nanocrystals is tuned from 4-11.0 $\mathrm{nm}$ by varying the reaction temperatures. The growth process and characterization of CdSe nanocrystals are determined by photoluminescence (PL) spectroscopy, ultraviolet-visible (UV-Vis) spectroscopy, X-ray photoelectron spectrometry (XPS), X-ray powder diffraction (XRD), transmission electron microscopy (TEM) and high-resolution transmission electron microscopy (HRTEM). The influence of reaction conditions on the growth of CdSe nanocrystals demonstrates that low reaction temperature is favourable for the formation of high quality CdSe nanocrystals.
\end{abstract}

Keywords. Nanomaterials; semiconductors; optical properties; defect formation.

\section{Introduction}

Research on colloid semiconductor nanocrystals has been a distinguishable topic in the field of modern nanoscale science and technology (Steigerwald and Brus 1990; Wang et al 2006). Among the various semiconductor materials, colloidal CdSe nanocrystals are the most actively studied owing to their size-dependent emission in the visible range (Weller 1993), the advances in their synthesis and their potential applications in various fields, such as light-emitting diodes (Lee et al 2000; Schaller et al 2006), lasers (Schlamp et al 1997; Artemyev et al 2001; Maskaly et al 2006), solar energy cell (Kornowski et al 1996; Suri and Mehra 2007) and biomedical tags (Bruchez et al 1998; Zhang et al 2003; Alivisatos et al 2005; Michalet et al 2005). In practice, such extensive applications have great potential to promote the synthesis of CdSe nanocrystals to the welldeveloped level. Among various traditional synthesis methods of nanomaterials, the most conventional route to synthesize the high quality CdSe nanocrystals is colloid chemistry: in the early 1990 s, dimethyl cadmium was used as the cadmium precursor to react with bis (trimethylsily)selenium in a coordinating solvent trioctylphosphine oxide (TOPO) (Murray et al 1993). Later on, Manna and co-workers (2000) have generally studied the influential factors in shape control of CdSe nanocrystals by changing the ratio of surfactants, injection amount, and time-dependent monomer concentration using

\footnotetext{
*Author for correspondence (zhangxl@bit.edu.cn)
}

dimethyl cadmium as the cadmium precursor. Recently, the morphologies and optical properties of $\mathrm{CdSe}$ nanocrystals have been systematically studied by directly using $\mathrm{CdO}$ or $\mathrm{Cd}(\mathrm{Ac})_{2}$ as cadmium precursor in different solvent systems, ratio of the precursor, injection rates and concentrations of the ligands (Peng and Peng 2001; Qu et al 2001; Peng 2002; Yu and Peng 2002).

However, the above studies discussed in detail the influential factors of CdSe nanocrystals synthesis except for reaction temperature. As part of the development of strategies for the synthesis of high quality colloidal nanocrystals, here we described the influence of the reaction temperature on colloidal CdSe nanocrystals synthesis at four different temperatures. The relationship between the optical properties of the as-prepared CdSe nanocrystals and a variety of growth conditions was also studied. Obtained CdSe nanocrystals were characterized by ultraviolet-visible (UV-Vis), photoluminescence (PL) spectra, X-ray photoelectron spectrometry (XPS), X-ray diffraction (XRD), transmission electron microscopy (TEM) and high resolution transmission electron microscopy (HRTEM) tomography.

\section{Experimental}

\subsection{Chemicals}

Se powder, stearic acid (SA), trioctylphosphine (TOP) and trioctylphosphine oxide (TOPO) were purchased from Alfa Aesar Chemicals and used without any purification. The solvents used and $\mathrm{Cd}\left(\mathrm{CH}_{3} \mathrm{CH}_{2} \mathrm{COO}\right)_{2} \cdot 2 \mathrm{H}_{2} \mathrm{O}$ were purchased from Chemical Reagent Corporation of Beijing. 


\subsection{Synthesis}

The synthesis of CdSe nanocrystals is similar to the methods reported by $\mathrm{Qu}$ and co-workers (2001). A typical synthesis is as follows. $\mathrm{Cd}(\mathrm{Ac})_{2} \cdot 2 \mathrm{H}_{2} \mathrm{O}, 0.0133 \mathrm{~g}(0.05$ $\mathrm{mmol}$ ), and $1.0000 \mathrm{~g} \mathrm{SA}$ were loaded into a $25 \mathrm{~mL}$ threeneck flask and heated to $120^{\circ} \mathrm{C}$ under nitrogen flow, after $\mathrm{Cd}(\mathrm{Ac})_{2} \cdot 2 \mathrm{H}_{2} \mathrm{O}$ was completely dissolved, the mixture was allowed to cool to room temperature, $1.4332 \mathrm{~g}$ TOPO was added to the flask, and the mixture was heated to 200 $280^{\circ} \mathrm{C}$ under argon flow to form an optically clear solution. At this temperature, the Se solution containing $0.0197 \mathrm{~g}(0.25 \mathrm{mmol})$ of Se dissolved in $0.8803 \mathrm{~g}$ $(2.38 \mathrm{mmol})$ TOP and $0.2 \mathrm{~g}$ toluene was swiftly injected into the reaction flask. After the injection, the temperature was set at $200-280^{\circ} \mathrm{C}$ for the growth of nanocrystals. At various time intervals, aliquots with a needle-tip amount of the reaction mixture were removed and diluted by toluene. Insoluble white solid, if it existed, was separated by centrifugation and decantation prior to further measurements.

\subsection{Characterization}

The size and shape of these CdSe nanocrystals were examined using transmission electron microscopy (TEM) and high resolution transmission electron microscopy (HRTEM). TEM and HRTEM images were obtained using Jeol-200CX (operating at $120 \mathrm{kV}$ ) and FET TECNAI F30 (operating at $200 \mathrm{kV}$ ), respectively. The sample for TEM was prepared by placing a drop of toluene dispersion of CdSe nanocrystals on the amorphous carbon-coated copper grids. The structure of the nanocrystals was investigated by X-ray diffraction (XRD) using a Rigaku D/MAX 2400 X-ray diffractometer with $\mathrm{CuK}_{\alpha}$ radiation $(\lambda=1.5405 \AA)$. Prior to the XRD measurements, the samples were prepared by spreading several drops of CdSe nanocrystals on the glass substrate. The surface of CdSe nanocrystals was measured by using a VG ESCALAB-5 X-ray photoelectron spectrometry (XPS) system. Optical absorption spectra were collected at room temperature on a PE Lambda 35 Ultravioletvisible (UV-Vis) spectrometer using $1 \mathrm{~cm}$ quartz cuvettes. The toluene solvent was used for the background sample. Room temperature photoluminescence (PL) spectra were carried out on a SPEX Fluorolog-2 spectrometer of front face collection with $500-\mu \mathrm{m}$ slits. PL spectra were collected between 400 and $700 \mathrm{~nm}$ at room temperature with $480 \mathrm{~nm}$ excitation energy.

\section{Results and discussion}

\subsection{Absorption analysis}

The growth kinetics of the nanocrystals was monitored by collecting samples from the reaction vessel at different reaction times. After injection of the Se solution, the nucleation and growth of CdSe nanocrystals occurred at different reaction temperatures. The formation process of CdSe nanocrystals can be identified from both the colour change and the UV-Vis spectra of the solution. As the reaction progressed, the solution was observed to be from colourless to transparent light yellow to orange yellow and then red brown, which directly indicated the formation of different sized CdSe nanocrystals. Figure 1 shows shifts of the peak positions and the shapes of the UV-Vis absorption spectra during the whole reaction process at four different temperatures $\left(200,220,240\right.$ and $\left.280^{\circ} \mathrm{C}\right)$. During the whole reaction process, growth of the particles was clearly evidenced by the shift of absorption spectra to longer wavelengths as a consequence of the quantum confinement effect. With the raise of reaction temperature, the growth rate of the CdSe nanocrystals increases. The width of the absorption band reflects the size distributions of the nanocrystals in the crude solutions. The sharp absorption features suggested highly monodisperse samples. A substantial difference in the growth evolution was observed between four growth temperatures.

At lower reaction temperature $\left(200^{\circ} \mathrm{C}\right)$, peak position of CdSe nanocrystals shifted to long wave when reaction time ranged from $2 \mathrm{~min}(495 \mathrm{~nm})$ to $15 \mathrm{~min}(531 \mathrm{~nm})$ (figure 1a). This result indicates that the size of $\mathrm{CdSe}$ nanocrystals gradually becomes larger with increase in reaction time. Similar results were obtained when the reaction temperature was increased to $220^{\circ} \mathrm{C}$, as shown in figure $1 \mathrm{~b}$. Interestingly, at higher reaction temperature $\left(240\right.$ or $280^{\circ} \mathrm{C}$, the absorption peak did not attain any shape at all, and this phenomenon lasted for the entire growth course (figures 1c, d). Specifically, when the reaction temperature was increased to $280^{\circ} \mathrm{C}$, the flat absorption peak ranged from $1 \mathrm{~min}(629 \mathrm{~nm})$ to $10 \mathrm{~min}$ $(649 \mathrm{~nm})$. This result revealed that the size of $\mathrm{CdSe}$ nanocrystals gradually increased as reaction time prolonged. Later on, very little change was observed for the position of the peak, and the wavelength located at $650 \mathrm{~nm}$ (15 min), indicating that the nucleation and growth process took $<10 \mathrm{~min}$ (figure 1d). At the same initial time of reaction ( $1 \mathrm{~min}$ ) in four reactions, the position of the absorption peak appeared at $495 \mathrm{~nm}\left(200^{\circ} \mathrm{C}\right), 533 \mathrm{~nm}$ $\left(280^{\circ} \mathrm{C}\right), 585 \mathrm{~nm}\left(240^{\circ} \mathrm{C}\right)$ and $629 \mathrm{~nm}\left(280^{\circ} \mathrm{C}\right)$, respectively. These observations can be explained as due to the relative rates of nucleation and growth at low temperatures being slower than at high temperature, if the absolute value of monomer concentration was same. Moreover, when compared to the results shown in figures $1 \mathrm{c}$ and $\mathrm{d}$, the sharper absorption peaks and smaller value of fullwidth at half maximum (FWHM) as shown in figures la and $b$, indicate that the size of CdSe nanocrystals can be close to monodisperse size distribution $(\mathrm{Qu}$ et al 2001). The possible reason is that high reaction temperature results in formation of irregular nanocrystals due to faster 

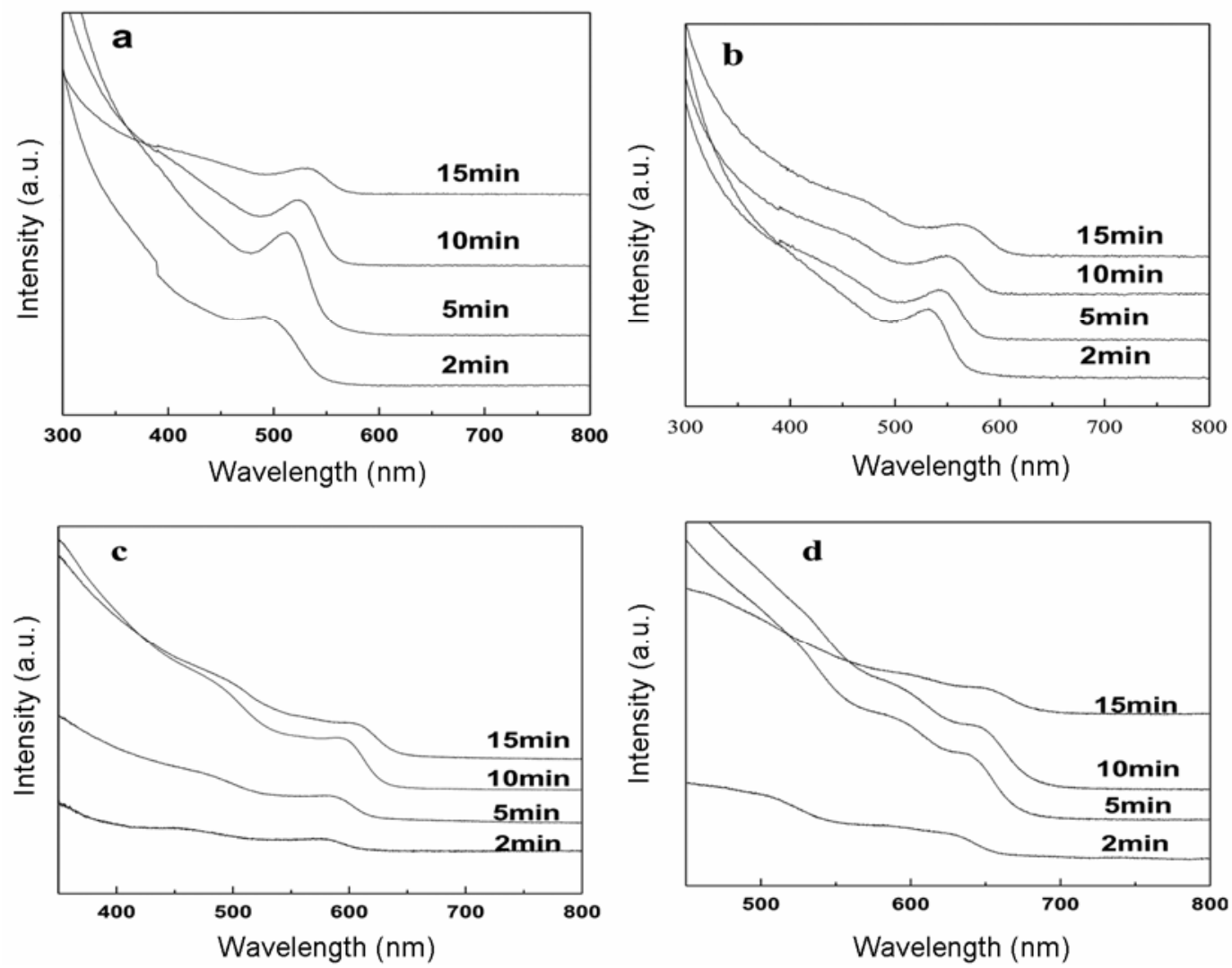

Figure 1. Temporal evolution of the absorption spectra of colloidal CdSe nanocrystals during their growth at different temperatures. a. $200^{\circ} \mathrm{C}$, b. $220^{\circ} \mathrm{C}$, c. $240^{\circ} \mathrm{C}$ and d. $280^{\circ} \mathrm{C}$.

nucleation and growth of nanocrystals. Taken together, these facts clearly suggest that the CdSe nanocrystals with desired size will form at the lower reaction temperature. Therefore, it is believed that the reaction temperature plays a major role in determining nucleation and growth of the as-synthesized CdSe nanocrystals.

\subsection{PL analysis}

Figure 2 indicates PL spectra of CdSe nanocrystals synthesized at different reaction temperatures. The PL spectrum is red-shifted compared to the absorption spectrum, which indicated the large Stokes shift of the nanocrystals. With the raise of the reaction temperature, the PL spectra occurrence dramatically changed. At lower reaction temperature $\left(200^{\circ} \mathrm{C}\right)$, the $\mathrm{PL}$ spectrum shows narrow FWHM value from reaction $5 \mathrm{~min}(32 \mathrm{~nm})$ to $15 \mathrm{~min}(36 \mathrm{~nm})$ as shown in figure $2 \mathrm{~A}$, which indicated growth of crystallites with few electronic defects sites. Similar results were obtained from $2 \mathrm{~min}(31 \mathrm{~nm})$ to $15 \mathrm{~min}(35 \mathrm{~nm})$ when the reaction temperature was increased to $220^{\circ} \mathrm{C}$ (figure $2 \mathrm{~B})$. At high reaction temperature $\left(240^{\circ} \mathrm{C}\right)$, there had been two exciton peaks at $560 \mathrm{~nm}$ and from $576 \mathrm{~nm}$ extended to the region of near infrared from reaction time of $2 \mathrm{~min}$ to $10 \mathrm{~min}$ (figure $2 \mathrm{C}$ ). The appearance of the first exciton peak around $560 \mathrm{~nm}$ originates from the band edge of the as-prepared CdSe nanocrystals. In addition to the excitonic emission, the broad band emission from $576 \mathrm{~nm}$ extended to the region of near infrared arises from the defect states of CdSe nanocrystals. When the reaction temperature was increased to $280^{\circ} \mathrm{C}$, there also emerged two exciton peaks at $559 \mathrm{~nm}$ and $650 \mathrm{~nm}$ (figure 2D). These weaker and broader emission spectra suggest a large percentage of defects are located at the surface of CdSe nanocrystals. Why does this phenomenon appear? Taking into account the kinetics of the particle formation, the reaction temperature can effect the surface configuration of the nanocrystals on the packing of surface ligands. The higher reaction temperature is in favour of the capping groups attaching and detaching from the surface of CdSe nanocrystals, which results in a faster growth rate and larger big miscellaneous nanocrystals cannot be perfectly passivated. While the lower reaction temperature may make the ligands to have no enough energy to shed from the surface, which is propitious to form well-proportioned nanocrystals. Thus, high quality CdSe nanocrystals were successfully prepared at low reaction temperature.

\subsection{TEM analysis}

The diameter and narrow size distribution of the crude CdSe nanocrystals synthesized in this work were also 

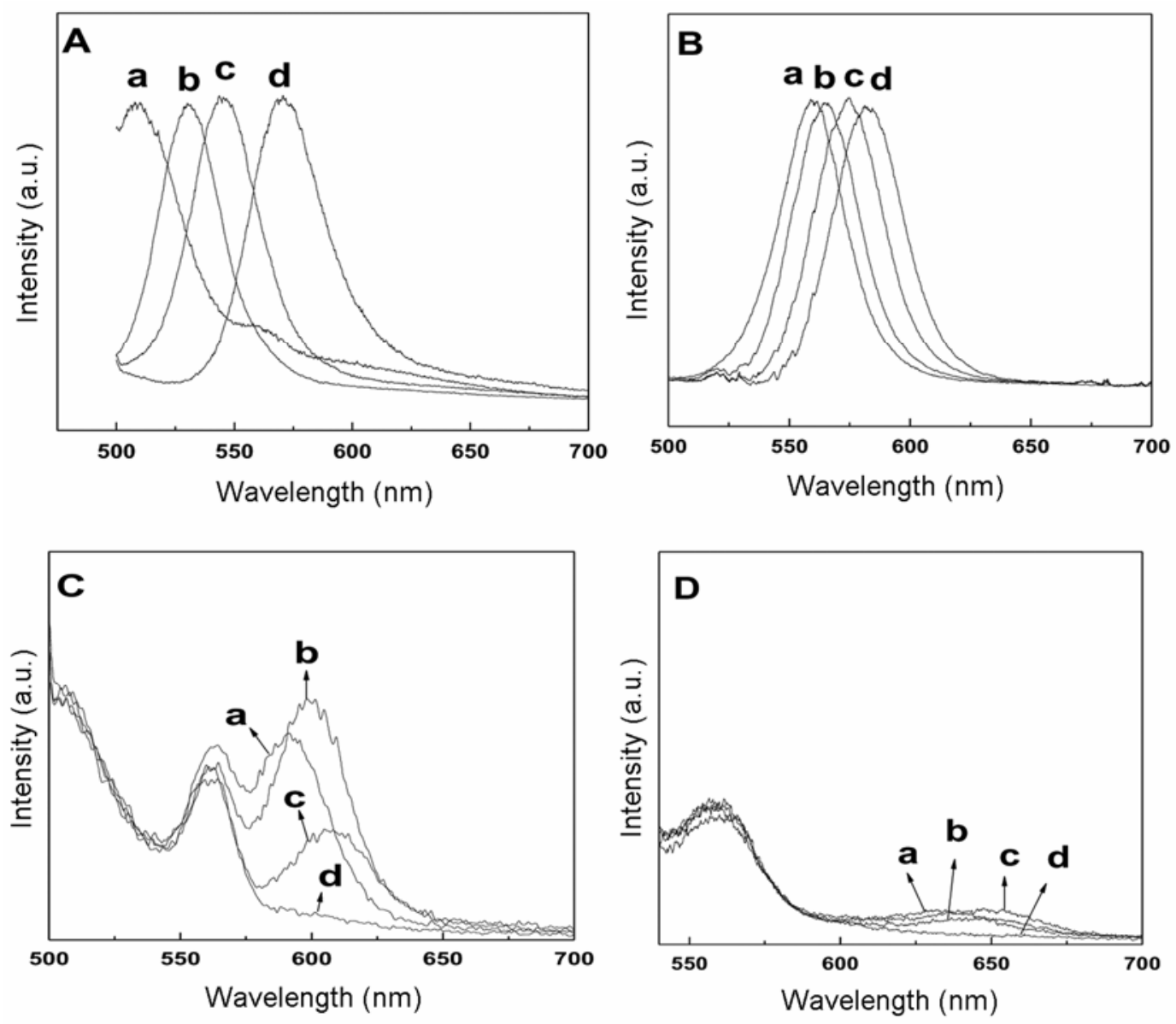

Figure 2. Photoluminescence spectra of colloidal CdSe nanocrystals during their different growth temperatures. A. $200^{\circ} \mathrm{C}$, B. $220^{\circ} \mathrm{C}$, C. $240^{\circ} \mathrm{C}$, D. $280^{\circ} \mathrm{C}$ and for different reaction times a. $2 \mathrm{~min}$, b. $5 \mathrm{~min}$, c. $10 \mathrm{~min}$ and d. $15 \mathrm{~min}$.

determined by TEM. With the raise of reaction temperature, diameter of CdSe nanocrystals becomes gradually big from $4 \cdot 5-11.0 \mathrm{~nm}$. A representative example was presented in figure 3 , for samples of CdSe nanocrystals grown at different temperatures for a reaction time of $15 \mathrm{~min}$. The average particle diameters were $4.5 \mathrm{~nm}$ at $200^{\circ} \mathrm{C}, 6 \mathrm{~nm}$ at $220^{\circ} \mathrm{C}$, and $9.8 \mathrm{~nm}$ at $240^{\circ} \mathrm{C}$, respectively. However, when the reaction temperature was raised to $280^{\circ} \mathrm{C}$, the products became a mixture of big nanocrystals $(60 \%)$ and small ones $(40 \%)$ as shown in figure $3 \mathrm{~d}$. When the reaction temperature was at $200^{\circ} \mathrm{C}$, the vague TEM image indicated the structure of $\mathrm{CdSe}$ nanocrystals to be incomplete owing to slower rates of nucleation and growth (figure 3a). While the reaction temperature was increased to $280^{\circ} \mathrm{C}$, the nonspherical CdSe nanocrystals (figure $3 \mathrm{~d}$ ) were formed due to faster nucleation and growth of nanocrystals. These results also corresponded to the XRD data analysis. The HRTEM image (inset of figure 3b) showed an ensemble of CdSe nanocrystals assembled into a locally well-ordered close-packed array. It could be observed that the nanocrystals were well cry- stallized, with interplanar distances of $3 \cdot 5 \AA$, which was consistent with the (111) planes of cubic CdSe (viz. $3 \cdot 510 \AA$ ). The crystal lattice appeared to be faultless in all the CdSe nanocrystals investigated.

\subsection{XRD analysis}

The crystallinity of the final products was detected by X-ray diffraction. Typical XRD patterns of the prepared CdSe nanocrystals (reaction time, $15 \mathrm{~min}$ ) at different reaction temperatures are shown in figure 4 . The three distinct diffraction peaks at $2 \theta=25.6^{\circ}, 42 \cdot 3^{\circ}$ and $49.7^{\circ}$ corresponded to the (111), (220) and (311) crystalline planes of cubic CdSe (JCPDS No. 19-191). The broadening of the diffraction peaks indicated the small size of the obtained CdSe nanocrystals, which was consistent with the result from the UV-Vis spectra and TEM. With the reaction temperature higher, the intensity of the XRD peaks increased, which may indicate that the reaction temperature has important influence on the crystallinity of CdSe nanocrystals. 

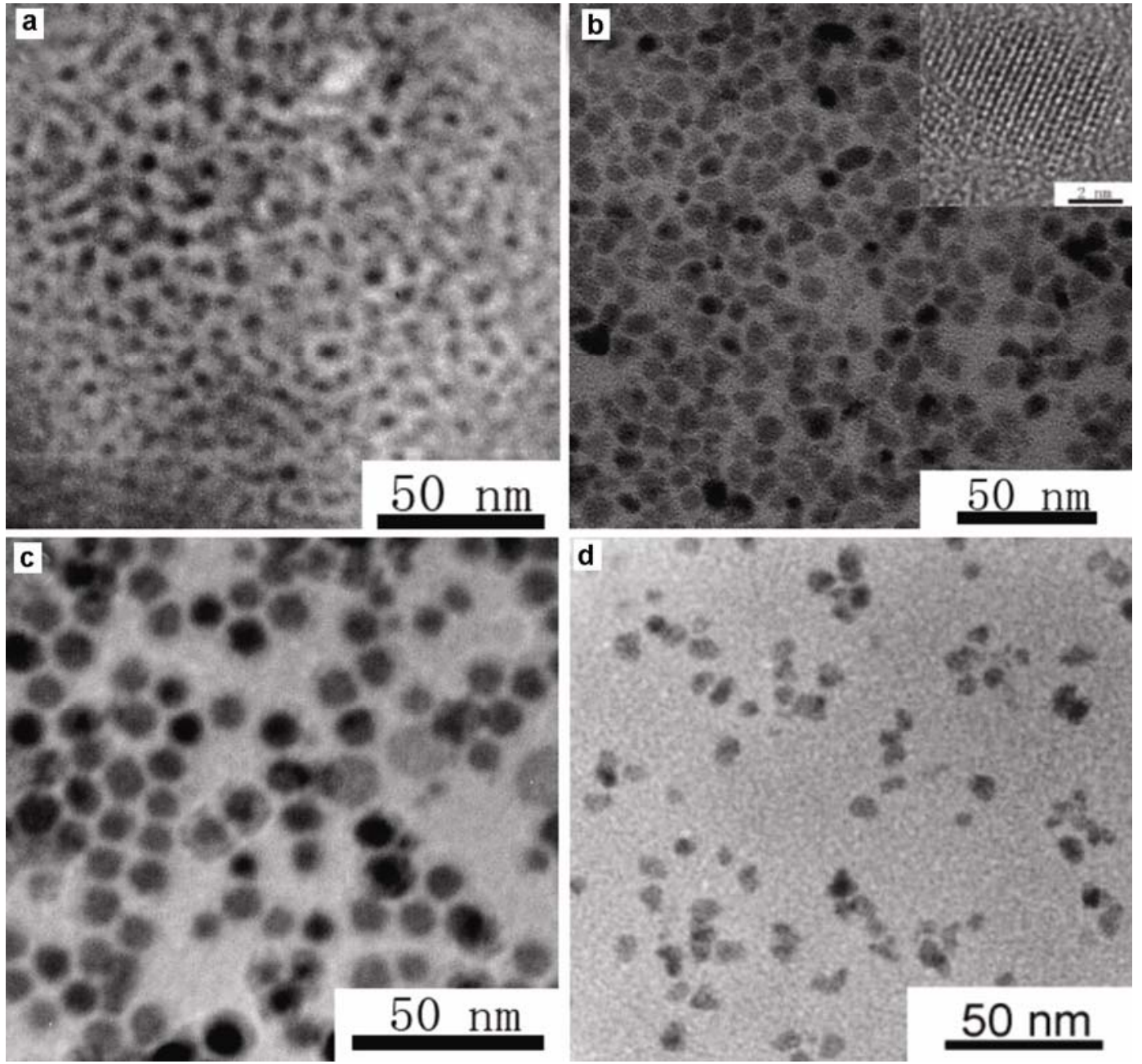

Figure 3. Typical TEM images of as-prepared CdSe nanocrystals under different reaction temperatures for reaction time of $15 \mathrm{~min}$ : a. $200^{\circ} \mathrm{C} ;$ b. $220^{\circ} \mathrm{C}$; c. $240^{\circ} \mathrm{C}$ and d. $280^{\circ} \mathrm{C}$. Inset shows HRTEM image corresponding to $220^{\circ} \mathrm{C}$.

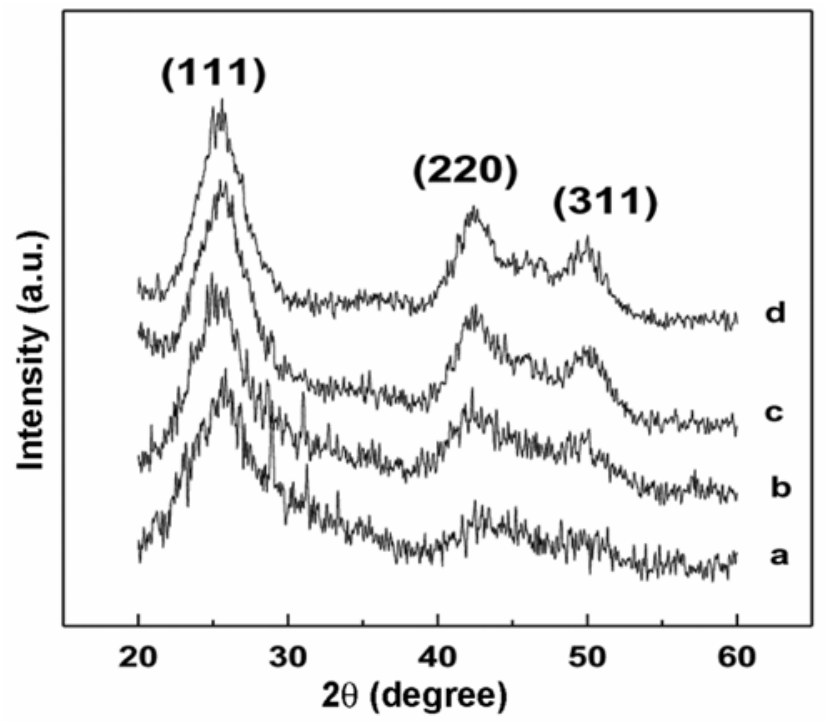

Figure 4. XRD patterns of CdSe nanocrystals obtained under different reaction temperatures for reaction time of $15 \mathrm{~min}$ : a. $200^{\circ} \mathrm{C} ;$ b. $220^{\circ} \mathrm{C} ;$ c. $240^{\circ} \mathrm{C}$ and d. $280^{\circ} \mathrm{C}$.

\subsection{XPS analysis}

The XPS spectra shown in figure 5 give further evidence for the purity of the product. All the peaks were calibrated by using $\mathrm{C}(1 s)(284.5 \mathrm{eV})$ as the reference. The two peaks located at $405.7 \mathrm{eV}$ and $412.7 \mathrm{eV}$ are assigned to $\mathrm{Cd}(3 d)$ and that at $58.0 \mathrm{eV}$ corresponds to $\mathrm{Se}(3 d)$. These results are close to those of the pure $\mathrm{CdSe}$. No impurities such as $\mathrm{SeO}_{2}, \mathrm{Cd}_{3} \mathrm{P}_{2}, \mathrm{CdO}$ were detected in the XPS analysis, which was consistent with the XRD results.

\section{Conclusions}

We systematically investigated effects of reaction temperature on the size and optical properties of CdSe nanocrystals. The reaction temperature has important influence on optical properties due to the surface configuration of the nanocrystals on the packing of surface ligands. The size of CdSe nanocrystals may increase with the raise of 



Figure 5. XPS spectra of organic molecule-capped CdSe nanocrystal obtained at $220^{\circ} \mathrm{C}$ for reaction time of $15 \mathrm{~min}$ : a. XPS full spectrum, b. Cd element and c. Se element. reaction temperature because of different rates of nucleation and growth. It has also been seen that the different reaction temperatures lead to $\mathrm{CdSe}$ nanocrystals synthesized with the zinc blende structure. The unique optical properties suggest a promising future of the nanocrystals in practical applications.

\section{Acknowledgements}

The work is supported by the 111 Project B07012. The authors would like to express sincere thanks to Prof. H J Gao and C M Shen, Institute of Physics (Chinese Academy of Sciences) for their assistance in experiments and helpful discussions.

\section{References}

Alivisatos A P, Gu W and Larabell 2005 Annu. Rev. Biomed. Eng. 755

Artemyev M V, Woggon U, Wannemacher R, Jaschinski H and Langbein W 2001 Nano. Lett. 1309

Bruchez Jr M, Moronne M, Gin P, Weiss S and Alivisatos A P 1998 Science 2812013

Kornowski A, Eichberger R, Giersig $M$, Weller $\mathrm{H}$ and Eychmuller 1996 J. Phys. Chem. 10012467

Lee J, Sundar V C and Heine J R 2000 Adv. Mater. 121102

Manna L, Scher E C and Alivisatos A P $2000 \mathrm{~J}$. Am. Chem. Soc. 12212700

Maskaly G R, Petruska M A, Nanda J, Bezel I V, Schaller R D and Htoon H 2006 Adv. Mater. 18343

Michalet X, Pinaud F F, Bentolila L A, Tsay J M, Doose S, Li J J, Wu A M, Gambhir S S and Weiss S 2005 Science 307538

Murray C B, Norris D J and Bawendi M G $1993 \mathrm{~J}$. Am. Chem. Soc. 1158706

Peng X G 2002 Chem. Eur. J. 8335

Peng Z A and Peng X G 2001 J. Am. Chem. Soc. 123183

Qu L H, Peng Z A and Peng X G 2001 Nano. Lett. 1333

Schlamp M C, Peng X G and Alivisatos A P 1997 J. Appl. Phys. 825837

Schaller R D, Sykora N, Pietryga J M and Klimov V I 2006 Nano. Lett. 6424

Steigerwald M L and Brus L E 1990 Acc. Chem. Res. 23183

Suri P and Mehra R M 2007 Sol. Ener. Mat. \& Sol. C. 91518

Wang F, Shan J, Islam M A, Herman I P, Bonn M and Heinz T F 2006 Nat. Mater. 5861

Weller H 1993 Angew. Chem. Int. Ed. Engl. 3241

Yu W W and Peng X G 2002 Angew. Chem. Int. Ed. 412368

Zhang H, Wang L P, Xiong H M, Hu L, Yang B and Li W 2003 Adv. Mater. 151712 